1 Patient editor, The BMJ

2 Patient editor, The BMJ

Cite this as: BMJ 2021;375:n2687 http://dx.doi.org/10.1136/bmj.n2687 Published: 4 November 2021

\section{Access to personal health records-why is progress so slow?}

\author{
As part of its Patient and Public Partnership Strategy, The $B M J$ is shining a spotlight on the barriers \\ and challenges to enabling better access to personal health records. The BMPs patient editors start \\ to unpick why progress has been so slow, and how to speed it up.
}

\author{
Emma Doble, ${ }^{1}$ Henry Scowcroft ${ }^{2}$
}

Accessing data about ourselves, for the most part, has never been easier. Personal data on our finances, our Twitter account, Facebook page, phone network, stocks and shares, browsing habits, broadband consumption... even the number of steps we take and the minutes' sleep we get, can now be logged and accessed. There is barely a corner of our lives left untouched by the data revolution.

The move to more data has also been seen in healthcare. This is particularly true in long term conditions like type 1 diabetes (T1D), where the amount of data available to patients has increased dramatically over the past 10 years. This has altered the way in which people living with T1D manage their condition day to day. It has also completely changed the doctor-patient relationship as patients are more informed about their condition and management. The power dynamic has shifted, which for most has been a very positive experience.

But when it comes to personal health records-what's in them, who can access them, when they were last updated-we seem to be in the relative dark-ages.

This is perplexing, particularly given phenomenal leaps in frontline medical technology for delivering care and treatment, and growing public interest in-and frustrations with-accessing particular health records such as covid-19 vaccine passports. ${ }^{1}$

There have also been advances in technology platforms and digital literacy, and favourable changes in legislation (notably in the United States). ${ }^{2}$ And it's not that there isn't solid evidence that accessing one's records is beneficial to patient and healthcare professional alike, as even a cursory skim of OpenNotes' research repository reveals. ${ }^{3}$

So why has progress been so slow?

For a start there are ethical concerns: worries that reading jargon-heavy notes may somehow harm patients, or that it may compromise trust between doctor and patient. Similarly, there are worries around patients' loss of autonomy, and around data security. But as Charlotte Blease recently wrote, the flip side-the ethics of not providing access, is seldom discussed. ${ }^{4}$

There are also professional and cultural concerns: worries that relationships between patients and doctors will be broken. What information can be shared and is there anything which shouldn't be? Who should determine this? There are concerns that difficult news will be shared through a computer without having a trained professional there to support or guide, and fears around governance-what information can be shared and who might be able to access this.

Others worry about the impact on health inequalities, and how to ensure that enabling patient access to records does not exacerbate pre-existing digital and other divides. Accessing health records is always going to require passwords and security. But ensuring patients who wish to use them can easily find and access their own records is paramount. Patients need to not only be able to access their health records but also-importantly-be able to understand and engage with them. On the security point, however, it should be noted that "traditional" paper records are harder to lock down, and can easily be left unattended in, say, a waiting room or taxi cab (as both of us writing this have experienced).

Finally, and most obviously, there are logistical, technical, and legal challenges. Implementing robust, scalable, secure technology platforms across complex healthcare systems, where multiple regional and national bureaucracies intersect and compete for oversight and governance, and multiple technology providers exist, is clearly a difficult task. In the UK there have been recent, and welcome, commitments from NHS England and NHSX to ensure GP practices that use differing systems arrive at the same endpoint eventually. ${ }^{5}$ But a similarly complex picture exists in other sectors-banking for example-and progress has been far more swift there.

All of these issues are important, but none should be insurmountable. The BMJ has long believed that all of us must have easy, real-time access to their medical records and notes. ${ }^{6}$ In an upcoming series of webinars, hosted by the BMJ Patient Editor team, a powerful line-up of international experts will explore the underlying reasons for this slow progress from a variety of angles. In doing so, we aim to stimulate debate, discussion-and hopefully, action. You can sign up for the webinar series here: https://linktr.ee/BMJPatient ${ }^{7}$

Competing interests: none declared

Provenance and peer review: commissioned, not peer reviewed

Scowcroft H. Left in limbo-barriers to covid "passports" for trial participants will deter people from taking part in research. BMJ Opinion. June 2021 https://blogs.bmj.com/bmi/2021/06/07/left-in-limbo-barriers-to-covidpassports-for-trial-participants-will-deter-people-from-taking-part-in-research/

2 Salmi L, Blease C, Hägglund M, Walker J, DesRoches CM. US policy requires immediate release of records to patients. BMJ 2021;372:n426. doi: 10.1136/bmj.n426. pmid: 33602667

3 https://www.opennotes.org/research/ 
4 Blease C. We need to talk about “closed notes”. BMJ Opinion. October 2021. https://blogs.bmj.com/bmj/2021/10/15/charlotte-blease-we-need-to-talk-about-closed-notes/

5 https://digital.nhs.uk/services/nhs-app/nhs-app-guidance-for-gp-practices/accelerating-patientaccess-to-their-record

6 https://www.bmj.com/content/patient-access-health-records

7 https://linktr.ee/BMJPatient 